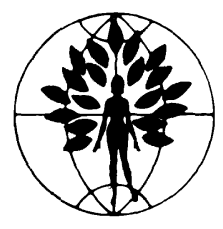

\title{
Screening for partner violence during pregnancy
}

\author{
E.M. Shadigian*, S.T. Bauer \\ University of Michigan Health System, Department of Obstetrics and Gynecology, Ann Arbor, MI, USA
}

Received 17 April 2003; received in revised form 10 July 2003; accepted 20 July 2003

\begin{abstract}
Objectives: An analysis of the international literature on partner violence provides insights for health care providers caring for women. Methods: The current literature on partner violence epidemiology, screening, and interventions was reviewed, focusing on pregnancy. Results: Adolescent girls and pregnant women are at highest risk for partner violence and homicide. Conclusion: Health care providers need to offer clinical screening and interventions to prevent consequences of partner violence, including homicide.

(C) 2003 International Federation of Gynecology and Obstetrics. Published by Elsevier Ireland Ltd. All rights reserved.

Keywords: Pregnancy; Domestic violence; Maternal mortality; Women's health; Adolescent

\section{Introduction}

Women continue to die worldwide at the hands of their intimate partners despite the commitment of medical professionals to screen for partner violence. Health care professionals continue to be asked to confront intimate partner violence in medical settings and to take sides in the conflict between victim and perpetrator, as it is morally impossible to remain neutral [1]. Batterers remain invisible and unacknowledged, but their criminal behaviors are obvious to their victims and the medical community. Victims seek medical care for short-term physical injuries and their long-term medical and psychological sequelae. The health

\footnotetext{
*Corresponding author. Tel.: +1-734-647-2021; fax: +1734-647-1006.

E-mail addresses:

eshadig@med.umich.edu (E.M. Shadigian),

sbauert@med.umich.edu (S.T. Bauer).
}

care community has the responsibility to provide excellent medical services, acknowledge the existence of abuse and identify cases, and be able to offer interventions. All health care professionals need to understand that, like racism and robbery, gender/partner violence is a behavior. The medical community is responsible for identifying victims, and for being a pivotal part of the effort to stop partner violence.

Partner violence refers to violence between individuals in an ongoing relationship, whether they live together or not, and regardless of sexual orientation. Misconceptions exist about who is likely to become a victim of intimate partner violence. Although the highest prevalence is found against young, poor, unmarried women, intimate partner violence traverses all demographic groups. Victims and perpetrators of intimate partner violence may be male or female and can be found in all age, racial, religious, socioeconomic, education-
\end{abstract}


al, and occupational groups. However, the victims of violence are predominantly women, and the perpetrators of violence predominantly men. Heterosexual women are 5-8 times more likely than heterosexual men to be victimized by an intimate partner. Men and women in homosexual relationships experience partner violence at a rate comparable to women in heterosexual relationships. Violence against women presents a multitude of forms. Health care providers often associate intimate partner violence with unwanted physical contact, assault, and common battery. However, they should be aware that it also includes such abuse as verbal threats, intimidation, coercion, economic abuse, isolation [2,6].

Pregnancy is a unique time when women tend to take greater interest in and responsibility for their own health and that of their children. Monthly and weekly prenatal visits may provide the greatest opportunity to screen for intimate partner violence. Providing tools to escape a violent partner benefits both mothers and their children.

\section{Epidemiology}

\subsection{General}

It was estimated in 1992 that at least 4 million women were annually assaulted by a male partner in the United States, but the Bureau of Justice Statistics recently reported that between 1993 and 2001, non-fatal violent crimes by intimate partners against women dropped by almost 50\% [3]. Nearly one-third of American women, $31 \%$, report having been physically or sexually abused by a husband or boyfriend at some point in their lives. Women who were abused as children are significantly more likely than other women to experience violence later in life. Sixty-six percent of the women who were abused as children report intimate partner violence, compared with $28 \%$ of those who did not experience child abuse [4].

Approximately $20 \%$ of married women have been forced to have sex during their marriages, and between 30 and $50 \%$ of all battered women are sexually assaulted [5]. In 2000 in the United States, approximately 1250 women were murdered by their current or former male partner [3]. It has been reported in 50 international population-based surveys that $10-50 \%$ of women experience intimate male partner violence, and that physical violence is accompanied by psychological abuse [5].

Pregnancy imparts no protection from violence. Partner violence rates are similar whether women pregnant are or not $[10,11]$; and it continues to claim lives, as four new studies in the past 2 years have demonstrated that homicide is the leading cause of maternal death in the United States [69].

\subsection{Intimate partner violence during pregnancy}

Prevalence rates of abuse during pregnancy are reported to be between 0.9 and $20.1 \%$. Studies that examined a variety of screening strategies revealed that higher prevalence rates are found when screening occurs: (1) more than once during pregnancy; (2) later in pregnancy (third trimester); and (3) when women are offered detailed interviews with trained professionals [10].

The alarming prevalence rates of physical abuse before and during pregnancy and during the postpartum period demonstrate that all women of reproductive age are at risk for intimate partner violence. Moreover, partner violence during a previous pregnancy strongly predicts a risk for future abuse. The highest estimated prevalence rates arise from screening over longer periods, when a rapport is built between victim and heath care provider and a support system is available [11].

Goodwin and colleagues [12] surveyed 39348 women in 14 states who were delivered of live offspring over a 2-year period. Of the women younger than 20 years, $18.5 \%$ experienced domestic violence during pregnancy, compared with $9.4 \%$ of the 20-29-year-old women and $4.4 \%$ of the women older than 30 years. Abused women have twice the chance of beginning prenatal care during the third trimester of pregnancy, and less prenatal care results in a potential increase in maternal and fetal complications. Less overall contact with the health care system diminishes the women's opportunity to be screened for partner violence and offered interventions and referrals. 
Table 1

Detrimental effects of partner violence during pregnancy

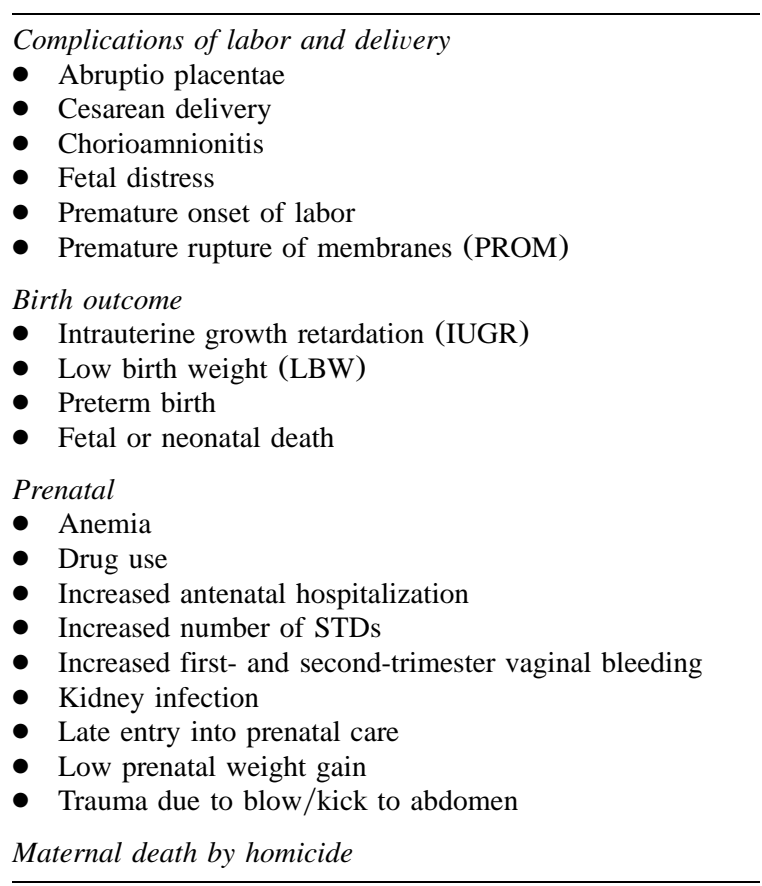

Attempted homicide, rape, physical abuse, and chronic partner violence during pregnancy are associated with multiple maternal and infant health problems (Table 1). Abused mothers are more likely to present with premature labor, enter prenatal care late, and have inadequate prenatal weight gain [6]. Rachana and colleagues [13] assessed 7105 pregnant women for physical violence over 3 years. The prevalence rate of physical violence, defined as a woman physically hurt by her husband or partner or involved with him in a physical fight during the 10 months before delivery, was $21 \%$. The authors demonstrated an association between maternal morbidity and physical violence during pregnancy. They identified several maternal conditions were that led to hospitalization before delivery and could be attributed to violence-these included kidney infection, premature labor, and abruptio placentae.

A systematic review and meta-analysis of 14 studies conducted in Canada found a significant association between abuse of pregnant women and low birth weight [14]. One study included in the meta-analysis, which had a sample size of more than 1000 pregnant women, established that partner violence and intimidation were associated with low birth weight (LBW) among women giving birth at term [15]. Studies of adolescent girls have shown that an abusive partner may pressure his girlfriend not to gain weight or seek health care [16]. Abuse may contribute to stress, which in turn has been associated with smoking and low weight gain, and consequently with low birth weight.

The rate of battered teenage girls who receive prenatal care only in the third trimester is $22 \%$, which increases the risk of LBW, compared with $7.5 \%$ for teens who are not battered. Additionally, even after accounting for other risk factors, battered pregnant women are up to four times more likely to have a low-birth-weight infant than nonbattered women [17].

\section{The effects of intimate partner violence on the health of female children and adolescents}

\subsection{Adolescent girls}

Sexual and physical violence are often connected for teenagers in abusive relationships. Pregnant teens often have a history of physical, sexual, and/ or other family-related violence. Those who have experienced childhood or adolescent sexual abuse also have greater sexual vulnerability, which increases the risks of unintended pregnancy and further abuse. Fergusson and colleagues [18] found that $62 \%$ of pregnant adolescents have been victims of molestation, attempted rape, or rape before their first pregnancy; and that $60 \%$ of pregnant adolescents with a history of sexual assault have been hit by a partner. In addition, $66 \%$ of infants born to adolescents have been fathered by men older than 18 years.

Many adolescents who have experienced childhood sexual assault do not believe that they can control what happens to them. They may have difficulty making choices preventing them from exposure to further abuse, and to unintended sexual consequences such as pregnancy or sexually transmitted diseases. Teens with a history of sexual assault are more likely to have had sexual intercourse before the age of 15 years, not to have 
used birth control at the last intercourse, to have had multiple sexual partners, and to become pregnant. Pregnancy occurs three times more often among adolescents who have been sexually assaulted than among those with no history of sexual assault [19].

A study of teenage mothers in Alaska revealed that they are more likely to experience violence during and after their pregnancy than older women. Research on partner violence tends to focus on violence during pregnancy, presumably because violence then affects fetal as well as maternal wellbeing. The Alaska article found that violence was more than two-fold higher during the postpartum period, which suggests that practitioners should extend their preventive efforts to that period [20].

Harrykissoon and colleagues [21] recently analyzed a multiethnic cohort of 540 young mothers who began having children as adolescents. The young women were screened for intimate partner violence at specific times $(3,6,9,12,18$ and 24 months) during the postpartum period. Of the mothers who reported partner violence during pregnancy, $75 \%$ also reported partner violence within 24 months following delivery. Partner violence prevalence was the highest 3 months postpartum (21\%), and the lowest 24 months postpartum $(13 \%)$. Harrykissoon concluded that adolescent girls are at high risk for experiencing partner violence during the postpartum period. Frequent screening of this young population by health care practitioners for partner violence is critical to maximize detection.

\subsection{Screening for child abuse and partner violence in the pediatrician's office}

At least half of all children whose mothers are battered are abused; thus, routine screening for child abuse is essential. Asking a question such as, "Are you worried that your kids are being hurt?" may be helpful in obtaining a true assessment of child abuse. Combining routine partner violence and child abuse screening at pediatric visits could accomplish improved detection. It may be necessary to involve child protective services. In 1998, the American Academy of Pediatrics issued a position statement stating that "the abuse of women is a pediatric issue," which makes a strong case for recognizing the consequences of partner violence in child health care settings.

Screening has been shown to be welcome by most parents, despite barriers to implement systemwide partner violence screening protocols. Parkinson and colleagues [22] reported on more than 500 mothers who were screened for domestic violence when they brought their children for wellchild visits during a 5-month period. More than $80 \%$ of the mothers in the study were in favor of such screening, and more than two-thirds did not recall being asked about domestic violence by any health care provider in the past.

With an estimated 3-10 million children exposed to violence between their parents each year, screening protocols have been developed for hospitals' emergency departments. A recent study detected four important areas of consideration when developing screening protocols for a pediatric emergency department. Trained screeners must: (1) demonstrate empathy, warmth, and a helpful attitude; (2) assess the child's medical needs first; (3) have an organized approach to detect the child's safety, and how and when it is appropriate to notify CPS when a parent screens positive for partner violence; and (4) routinely offer community resources [23].

\subsection{Homicide/maternal mortality}

Elaine Alpert, M.D., M.P.H. has estimated that if the 100-fold decrease seen in maternal mortality since the 19th century is to be matched by a similar decrease in the $21 \mathrm{st}$, the primary cause of fatality-murder at the hands of intimate partners-needs to be addressed for the sake of the health, safety, and wellbeing of women and children, and therefore society. The scope of maternal homicide is under-recognized, partially because the pregnancy status is not analyzed in studies on homicide or suicide. Currently, it is not known if women who experience partner violence are more or less at risk for homicide during pregnancy; the national Federal Bureau of Investigation statistics do not specify if a woman was pregnant at the time of the homicide [6]. 
Large urban and statewide studies have demonstrated that trauma is the leading cause of pregnancy-associated death, which is defined as all deaths from any cause during pregnancy or up to 1 year after pregnancy [24]. Dietz and colleagues [25] noted that $50 \%$ of the identified postpartum deaths by injury were homicides, and that the risk of homicide during postpartum among teenagers was 2.6 times greater than among non-postpartum teenagers.

In a retrospective cross-sectional study published in JAMA in 2001, Horon and Cheng [9] reviewed 247 pregnancy-associated deaths in Maryland between 1993 and 1998. Of these pregnancy-associated deaths, 50 (20\%) were by homicide. The study concluded that pregnant women are more likely to die from murder than from any other cause. However, women who die from homicide during pregnancy are often not included in maternal mortality statistics.

Based on reviews of 651 autopsy charts of women aged between 15 and 50 years who died during an 8-year period in Washington, DC, Krulewitch et al. [8] found that $43.4 \%$ of the murders of pregnant women were not reported, or that pregnancy was not mentioned in their death certificates.

A recent landmark study [6] described a definite link between abuse during pregnancy and attempted/completed homicide. The case-control study consisted of 437 cases of attempted/completed homicide (cases) against pregnant women and 384 cases of abused women (controls) living in the same area. Attempted and completed homicide were combined after analysis, and it was concluded that the two groups were not significantly different $(P<0.01)$. After adjusting for demographics, the authors found that the risk of becoming victim of attempted/completed homicide was three-fold higher for women abused during pregnancy.

\section{Screening and clinical management of inti- mate partner violence}

\subsection{Asking about abuse in a clinical setting}

Before practitioners can ask about abuse in a health care environment, they must define and understand the problem. Abuse in any form is a way of asserting power and control. Battering is not only a punch or kick. It involves dominating the victim emotionally, physically, sexually, and financially. Behaviors include pushing, shoving, punching, slapping, kicking, choking, assaulting with a weapon, restraining, leaving a person in a dangerous place, or refusing to help the sick or injured person. Before practitioners can competently ask questions about violence in a clinical arena, they must be their own family historians; they must explore their own experiences to be able to gauge their reactions toward patients in an office setting. The concepts of transference and countertransference are crucial to self-exploration.

Screening should be conducted by a health care provider who has been educated about the dynamics of intimate partner violence, the safety and autonomy of abused patients, and the necessity of culturally competent care. The trained physician or nurse should be able to provide information about intimate partner violence and local community resources, and intervene on behalf of identified victims. They should screen patients for current and lifetime victimization by intimate partner violence, and ask direct questions about physical, emotional, and sexual abuse. All adolescents and adult patients, regardless of cultural background, should be routinely screened, as well as parents and caregivers of children-regardless of the presence or absence of indicators of abuse.

Key elements in taking a comprehensive history include: (1) stating framing questions and asking direct questions in an empathetic way; (2) honoring privacy and always interviewing a patient in private; (3) screening for violence routinely. Routine screening for intimate partner violence and sexual assault can be accomplished while taking the social history. After asking about habits, such as cigarette, alcohol, and drug use and breast selfexam, in the same tone of voice and without changing inflection, tone, or volume, the practitioner should ask the recommended screening questions listed in Table 2.

\subsection{Interventions}

If the patient answers yes to a screening ques- 
Table 2

Screening for abuse: intimate partner violence and sexual assault screening guidelines

Screening can be conducted by starting with a framing statement followed by direct questions.

(Framing Statement)

"Because violence is so common, I now ask every woman about if she has experienced violence."

(Direct Question)

1. "Have you ever been in a relationship where you have been punched, kicked, choked, or hurt in any way?"

2. "Has anyone ever touched you inappropriately or forced you to have sex?"

tion, then several interventions should follow. A safety and legal assessment must be made, and health care providers should know their state and local laws about mandatory reporting for child abuse, elder abuse, and intimate partner violence. A thorough medical assessment includes documenting a history of partner violence and sexual assault. In addition, checking for traumatic marks or scars must be performed over the victim's entire body, and injuries documented visually using body maps and photographs - documentation will alert medical professionals of abuse and be helpful if used in a court of law. The provider should document her statements and avoid pejorative or judgmental documentation (e.g. write "patient declines services" rather than "patient refuses services"). Empathy is the key to a successful history and physical exam. A psychiatric assessment should be conducted that includes screening for depression, post-traumatic stress disorder (battered woman's syndrome), drug and alcohol use, child abuse, and risk of suicide or death from homicide. Former medical records should be obtained and patterns of violence and abuse appreciated.

Facilitated referrals offer immediate, direct help to victims by allowing them to make private phone calls in the safety of a private medical office. The health care provider may call an agency for the patient, put the patient on the phone, and leave the room. Shelter, legal advocacy, law enforcement, social workers, support groups, and therapy referrals can be very helpful.

Health care providers should assist women in determining their potential risk of death from homicide risk by using a danger assessment scale, which consists of 17 items in a yes/no response format. All items refer to risk factors that have been associated with murder in situations involving abuse [6].

Examples of the questions include asking about weapons, threats to kill, and increasing severity and frequency of abuse.

Finally, safety planning is essential to allow women to establish the safeguards needed before leaving, and the important documents and other personal items they need for themselves and their children.

Sometimes the most challenging patients to help are those who decide to stay in battering relationships while they contemplate leaving. The key to competent medical care in this situation is a nonjudgmental attitude. Instead of asking a patient why she stays, or merely telling her that she should leave, the health care provider should ask about her fear of death or severe harm, economic issues, cultural and religious beliefs, and how the legal system is helping or hindering her efforts. Psychological factors such as low self-esteem or the belief that she can change the perpetrator maybe part of her difficulty to leave. Having information about partner violence community advocacy programs and educational resources in bathrooms, waiting rooms, and private medical examination rooms sends a powerful message of no tolerance for violence by the health care community.

\section{Conclusions and new concepts}

Homicide is the leading cause of pregnancyassociated mortality. If women are abused during pregnancy, the rate of attempted/completed homicide against them is three times higher than the rate for other abused women. Disclosure of recent 
or ongoing intimate partner violence establishes an urgent or even emergent diagnosis, with intervention preventing death in many situations. The solidarity of health care professionals who routinely ask about intimate partner violence and create a protected space where survivors can safely disclose their experience ensures that perpetrators and their violent behaviors will no longer remain invisible and tolerated.

Physical and sexual violence has far-reaching medical and psychological ramifications because diseases may have higher incidence and prevalence rates among victims of violence. A radical shift needs to occur to create a new paradigm of human pathology. Otherwise, health care providers may remain intent on describing violence and trying to offer aid to victims, but miss the degree to which society must hold batterers accountable for contributing to the prevalence of many diseases and early deaths.

\section{References}

[1] Herman J. Trauma and recovery. 2nd ed. New York: Basic Books, 1997.

[2] Shadigian E. Domestic violence: identification and management for the clinician. Compr Ther 1996;22:424428.

[3] Rennison CM. Intimate partner violence, 1993-2001. US Department of Justice. Office of Justice Programs. Bureau of Justice Statistics, Crime Data Brief. NCJ 197838, 2003.

[4] Plichta S, Falik M. Prevalence of violence and its implications for women's health. Womens Health Issues 2001;11:244-258.

[5] Population Information Program, Center for Communication Programs, The Johns Hopkins University School of Public Health. Ending violence against women. Popul Rep 1999;27:1-44.

[6] McFarlane J, Campbell JC, Sharps P, Watson K. Abuse during pregnancy and femicide: urgent implications for women's health. Obstet Gynecol 2002;100:27-36.

[7] Granja AC, Zacarias E, Bergstrom S. Violent deaths: the hidden face of maternal mortality. $\mathrm{Br} \mathrm{J}$ Obstet Gynaecol 2002;109:5-8.

[8] Krulewitch CJ, Pierre-Louis ML, de Leon-Gomez M, Guy R, Green R. Hidden from view: violent deaths among pregnant women in the District of Columbia, 1988-1996. J Midwifery Women's Health 2001;46:410.
[9] Horon IL, Cheng D. Enhanced surveillance for pregnancy-associated mortality: Maryland, 1993-1998. J Am Med Assoc 2001;285:1455-1459.

[10] Gazmararian JA, Lazorick S, Spitz AM, Ballard TJ, Saltzman LE, Marks JS. Prevalence of violence against pregnant women. J Am Med Assoc 1996;275:19151920.

[11] Martin SL, Mackie L, Kupper LL, Buescher PA, Moracco KE. Physical abuse of women before, during, and after pregnancy. J Am Med Assoc 2001;285:15811584.

[12] Goodwin MM, Gazmararian JA, Johnson CH, Gilbert BC, Saltzman LE. The PRAMS Working Group. Pregnancy intendedness and physical abuse around the time of pregnancy: findings from the pregnancy risk assessment monitoring system, 1996-1997. Maternal Child Health J 2000;4:85-92.

[13] Rachana C, Suraiya K, Hisham AS, Abdulaziz AM, Hai A. Prevalence and complications of physical violence during pregnancy. Eur J Obstet Gynecol Reprod Biol 2002;103:26-29.

[14] Murphy CC, Schei B, Myhr TL, Du Mont J. Abuse: a risk factor for low birth weight? A systematic review and meta-analysis. Can Med Assoc J 2001;164:15671572.

[15] Campbell J, Torres S, Ryan J, King C, Campbell DW, Stallings RY, et al. Physical and nonphysical partner abuse and other risk factors for low birth weight among full term and preterm babies: a multiethnic case-control study. Am J Epidemiol 1999;150:713-726.

[16] Renker PR. Physical abuse, social support, self-care, and pregnancy outcomes of older adolescents. J Obstet Gynecol Neonatal Nurs 1999;28:377-388.

[17] Parker B, McFarlane J, Soeken K. Abuse during pregnancy: effects on maternal complications and birth weight in adult and teenage women. Obstet Gynecol 1994;84:323-328.

[18] Fergusson D, Horwood L, Lynskey M. Childhood sexual abuse, adolescent sexual behaviors and sexual revictimization. Child Abuse Negl 1997;21:789-803.

[19] Fiscella K, Kitzman H, Cole R, Sidora K, Olds D. Does child abuse predict adolescent pregnancy? Pediatrics 1998;101:620-624.

[20] Gesser BD, Perhman-Hester KA. Experience of violence among teenage mothers in Alaska. J Adolesc Health 1998;22:383-388.

[21] Harrykissoon SD, Rickert VI, Wiemann CM. Prevalence and patterns of intimate partner violence among adolescent mothers during the postpartum period. Arch Pediatr Adolesc Med 2002;156:325-330.

[22] Parkinson GW, Adams RC, Emerling FG. Maternal domestic violence screening in an office-based pediatric practice. Pediatrics 2001;108:E43.

[23] Dowd MD, Kennedy C, Knapp JF, Stallbaumer-Rouyer J. Mothers' and health care providers' perspectives on screening for intimate partner violence in a pediatric 
emergency department. Arch Pediatr Adolesc Med 2002;156:794-799.

[24] Dannenberg A, Carter DM, Lawson HW, Ashton DM, Dorfman SF, Graham EH. Homicide and other injuries as causes of maternal death in New York City, 1987 through 1991. Am J Obstet Gynecol 1995;172:15571564.
[25] Dietz PM, Rochat R, Thompson BL, Berg CJ, Griffin GW. Differences in the risk of homicide and other fatal injuries between postpartum women and other women of childbearing age: implications for prevention. Am J Public Health 1998;88B:641-643. 